\title{
Modeling and experiment validation of thermal control for the heat-stop of the Chinese Large Solar Telescope
}

\author{
Zhiwu Yan, ${ }^{\text {a,b,c }}$ Naiting Gu $\odot$, a,b,* Yangyi Liu, ${ }^{\mathrm{d}}$ Youming Guo, ${ }^{\text {a,b }}$ and \\ Changhui Rao ${ }^{\text {a,b,c, }, *}$ \\ ${ }^{a}$ Chinese Academy of Sciences, Key Laboratory on Adaptive Optics, Shuangliu, \\ Chengdu, Sichuan, China \\ ${ }^{\mathrm{b}}$ Chinese Academy of Sciences, Institute of Optics and Electronics, Chengdu, China \\ ${ }^{c}$ University of Chinese Academy of Sciences, Beijing, China \\ ${ }^{\mathrm{d}}$ Sichuan Police College, Luzhou, China
}

\begin{abstract}
Thermal control system is one of the most important components of large groundbased solar telescope. Heat-stop is one of the key components for controlling thermal effects for a large solar telescope, which reflects over $95 \%$ of solar radiation protecting the relay optics and post-focus instruments. Meanwhile, a part of solar radiation has also converted into thermal energy, which will lead to internal seeing effect. Thus, the thermal control for heat-stop is a necessary work. We deduce a mathematical model to express the relationship between thermal response of heat-stop and its structure design, ambient environment, and coolant input. We also experimentally validate the feasibility of the proposed method based on the Chinese Large Solar Telescope, which is the largest solar telescope in China. The experimental results show that the temperature difference between the experimental results and calculated results based the proposed model is $<2^{\circ} \mathrm{C}$. Moreover, the temperature difference between the surface temperature of the heat-stop and the ambient temperature can satisfy thermal control requirement of $\pm 5^{\circ} \mathrm{C}$. Therefore, it can be verified that our model is correct and consistent with the actual situation. (C) The Authors. Published by SPIE under a Creative Commons Attribution 4.0 Unported License. Distribution or reproduction of this work in whole or in part requires full attribution of the original publication, including its DOI. [DOI: 10.1117/1.JATIS.7.3.038003]
\end{abstract}

Keywords: heat-stop; solar telescope; mathematical model; experiment validation; Chinese Large Solar Telescope; thermal control.

Paper 20155 received Oct. 13, 2020; accepted for publication Jul. 15, 2021; published online Aug. 6, 2021.

\section{Introduction}

To answer the basic scientific questions in solar physics and space weather, the international community that operates in heliophysics research is designing future generation telescopes with ever greater diameter (e.g., Refs. 1 and 2). As the diameter increases, the spatial resolution increases, but also the photon budget on the primary focus and toward the transfer optics. As a consequence, it will bring serious thermal load to the thermal control system of the ground-based large aperture solar telescope. In serious cases, mirror seeing will be produced on the surface of the primary mirror and internal seeing effect will be produced at the heat-stop, which will seriously affect the observation ability of the large-aperture solar telescope. ${ }^{3,4}$ Therefore, the active thermal control system of the primary mirror and heat-stop must be designed for large-aperture ground-based solar telescope. Compared with the primary mirror, the heat-stop receives more solar radiation power per unit area, which makes it more difficult to control. Therefore, it is necessary to design a reasonable heat-stop structure and take away the absorbed solar radiation energy by liquid cooling, which can keep the surface temperature of the heat-stop consistent with the ambient temperature, so as to reduce the internal seeing effect and improve the imaging quality of the telescope.

*Address all correspondence to Naiting Gu,gnt7328@163.com; Changhui Rao, chrao@ioe.ac.cn 
The heat-stop is the core of the whole thermal control system. According to the requirements of the optical system configuration, the heat-stop has two different kinds of structure design, namely absorption type and reflection type. The off-axis Gregory optical system mainly adopts the way of thermal absorption, such as 1.6-m Goode Solar Telescope (GST) ${ }^{5}$ and Daniel K. Inouye Solar Telescope (DKIST) ${ }^{2,6-9}$ in America. While due to limited installation space, the telescope of the on-axis Gregory optical system mainly adopts the thermal reflection structure, such as German 1.5-m GREGOR Solar Telescope, ${ }^{10-13}$ European Solar Telescope (EST), ${ }^{1,14-16}$ and 1.8-m Chinese Large Solar Telescope (CLST). ${ }^{3,4,17-21}$

Through the study of the active temperature control system of the heat-stop of large aperture ground-based solar telescopes in the world, we find that the analysis method adopts CFD technology. GREGOR solar telescope mainly adopts single inlet dual cycle cooling structure based on the design of cooling structure. From the engineering point of view, it is qualitatively proposed that the maximum temperature of the heat-stop surface should not be higher than the ambient temperature of $5^{\circ} \mathrm{C} .{ }^{10-13} \mathrm{EST}$ solar telescope adopts flat plate reflection structure on the heat-stop structure and analyzes the air field temperature around the heat-stop by ANSYS software. Based on engineering experience, it is proposed that the maximum temperature on the heat-stop surface should be kept no higher than the ambient temperature of $8^{\circ} \mathrm{C} .{ }^{14-16}$ GST solar telescope adopts the cooling structure of the surface reflection and internal absorption on the heat-stop structure, and the thermal control requirement is that the surface temperature should not be $1^{\circ} \mathrm{C}$ higher than the ambient air. ${ }^{5}$ In the design of heat-stop structure of DKIST solar telescope, the circular rib structure is adopted on the absorption surface. From the perspective of engineering experience, it is proposed that the maximum temperature of the heat-stop surface should not be higher than the ambient temperature of $6{ }^{\circ} \mathrm{C}^{6-9}$ (the GREGOR is an on-axis Gregorian, whereas the DKIST is an off-axis Gregorian). The CLST adopts on-axis Gregory optical, in the beginning, CLST solar telescope in China is also based on the heat-stop structure, using a single cavity double inlet cooling structure. Through the fluid structure coupling analysis, it is proposed that the maximum temperature on the surface of the heat-stop should not be greater than $5^{\circ} \mathrm{C}$ of the ambient temperature..$^{3,4,17-21}$

Heat-stop is a thermal field stop, which can contain heat-rejecter or heat-trap and so on. They are all a form of heat-stop. To achieve real-time temperature control, it must be able to adjust the controller parameters according to the changes of the external environment to make the surface temperature of the heat-stop consistent with the ambient temperature. For the temperature control system of heat-stop, by establishing an accurate mathematical model of the heat-stop, the subsequent heat-stop temperature control system can be analyzed and designed, including the system stability and the selection of controller parameters. However, we have not found relevant papers describing mathematical model and the corresponding control strategy on thermal control of heat-stop.

This work specifies the model used to describe the thermal control system of the CLST heat rejecter and its experimental verification. The proposed model can provide a theoretical reference for the subsequent optimization of heat-stop structure and the implementation of temperature control strategy. The main arrangement of this paper is as follows: Sec. 2 mainly introduces the basic principle of thermal control of heat-stop and discusses the structure and cooling mode of heat-stop. Section 3 elaborates the law of conservation of energy, establishes its mathematical model for reflective heat-stop, and analyzes the main factors affecting the surface temperature of heat-stop. Section 4 combines with the corresponding experimental platform to verify the model of heat-stop through experiments. In Sec. 5, through analyzing the error of the model, the direction of improving the heat-stop is pointed out.

\section{Basic Principle of Thermal Control for Heat-Stop}

The heat-stop is a main subsystem of all large solar telescopes, even space ones, e.g., HINODE ${ }^{22}$ or ADAHELI. ${ }^{23}$ The heat-stop is the core component of the ground-based large-aperture solar telescope. It is located at the primary focus of the solar telescope and acts as the first field stop of the optical system. Here, due to the convergence of sunlight, the thermal load per unit area is very high, which will quickly heat the air around the heat-stop and produce the internal seeing 


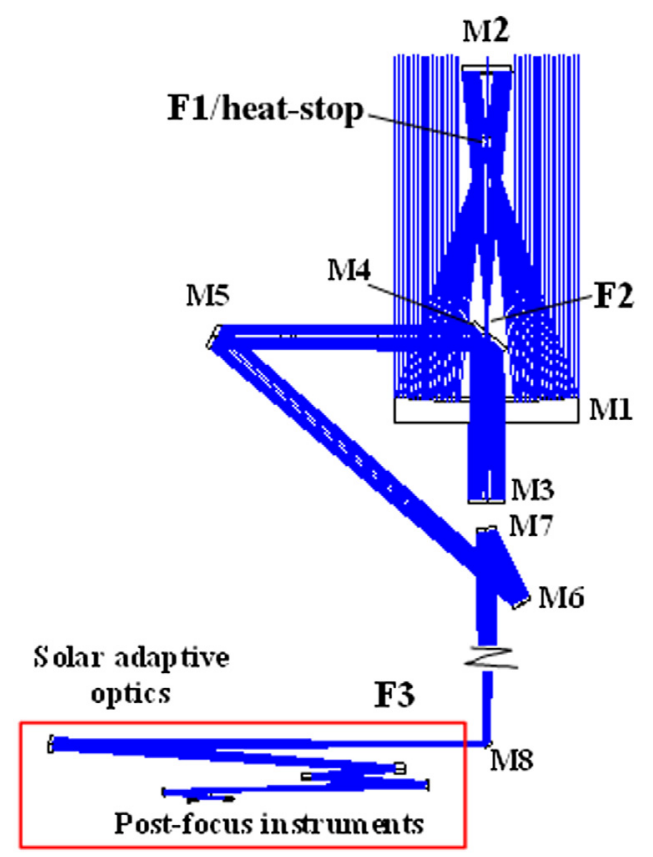

Fig. 1 The optical system of CLST.

effect. $^{3,4}$ The optical system of CLST is shown in Fig. 1. Through the thermal analysis of the system, the solar radiation energy mainly impacts the primary mirror and heat-stop, and the solar radiation power received by them is more than $2000 \mathrm{~W}$, as shown in Fig. 2. In fact, the load of solar radiation on M1 surface is distributed over the entire mirror, whereas in F1 it is concentrated. The corresponding power densities of solar radiation on M1 and HS surface are about 1200 and $3.2 \mathrm{MW} / \mathrm{m}^{2}$, respectively. ${ }^{3}$ Therefore, the active thermal control system must be used to keep the temperature of the heat-stop surface consistent with the ambient temperature. However, the temperature difference will change with the change of the external environment. For CLST, the acceptable temperature difference is within $\pm 5^{\circ} \mathrm{C}$.

The heat-stop thermal control system of the ground-based solar telescope is mainly composed of heat-stop, pipeline, electrical heater, refrigerator, electrical control valve, PLC, etc. The heat-stop thermal control scheme is shown in Fig. 3.

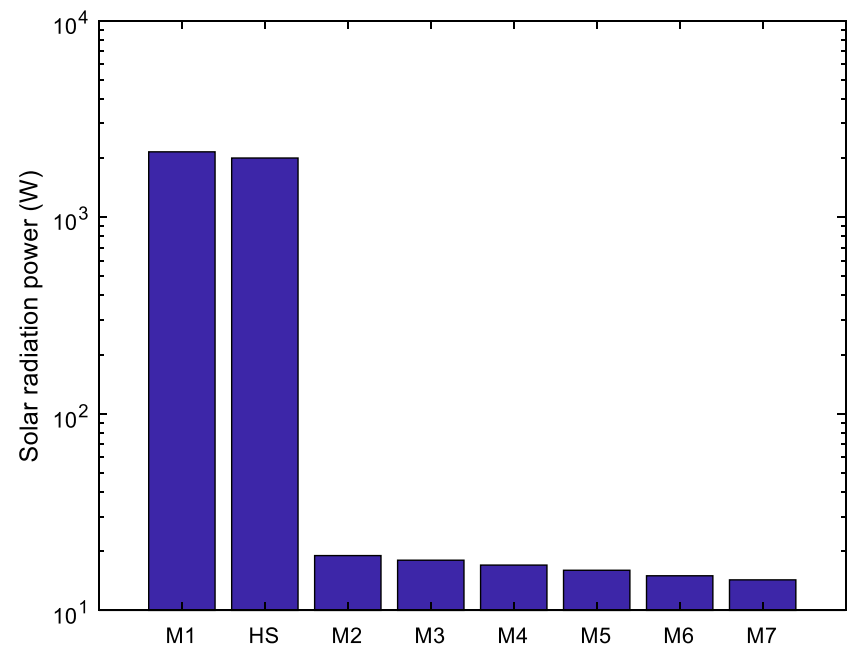

Fig. 2 Solar radiation power received by each optical surface of CLST. 


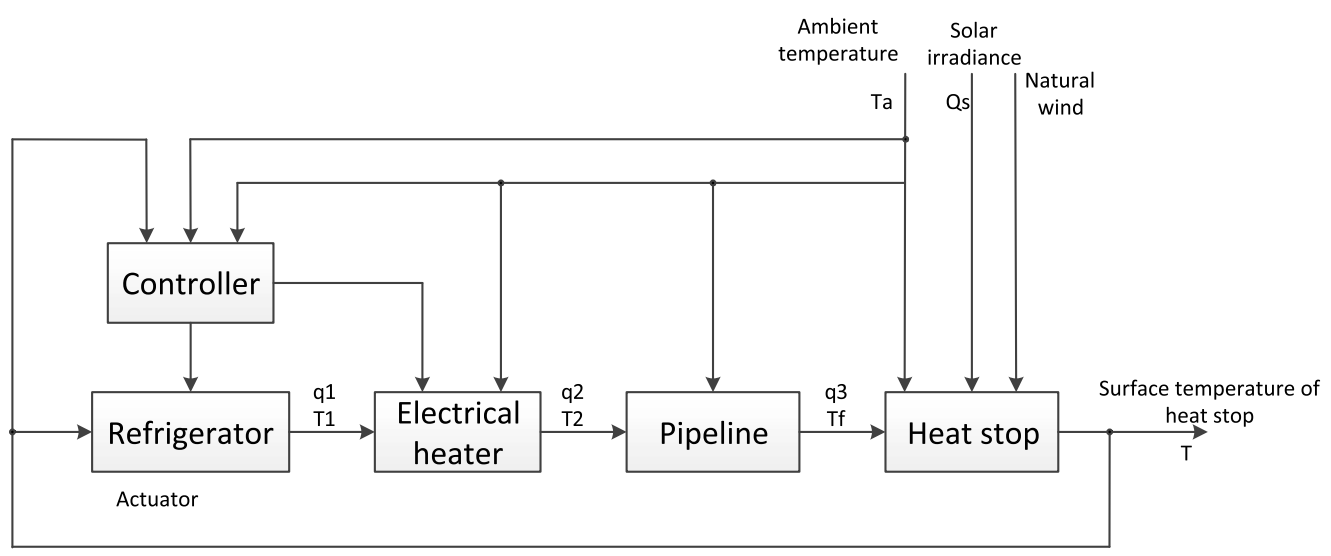

Fig. 3 The heat-stop thermal control scheme.

\section{Theoretical Modeling of Heat-Stop}

The heat-stop with thermal reflection structure is shown in Fig. 4. Protected silver coating is adopted on the reflective surface of heat-stop in the CLST. In fact, we expect the highest reflectivity up to $100 \%$, which means lower heat load at heat-stop. However, protected silver coating is a relatively good choice in practice by balancing feasibility, age, and cost. The comprehensive reflectivity of the protected silver coating is about 95\%. In the CLST system, thin $\mathrm{SiO}_{2}$ layer is coated over silver coating to reduce aging. At the same time, because the heat-stop absorbs part of the heat, its temperature will rise rapidly. For the body of the heat-stop, Glidcop copper alloy material with high thermal conductivity is adopted, whose thermal conductivity is $401 \mathrm{~W} / \mathrm{m} \cdot \mathrm{K}$. At the same time, a cooling liquid circulation system with double inlet and single cavity is used in the body to take away the heat absorbed by the heat-stop, so as to realize the active thermal control of the surface temperature of the heat-stop., ${ }^{3,4,17,18}$

Without an active temperature control system, the surface temperature of the heat-stop at the primary focus will rise rapidly, and the temperature difference between the surface temperature of the heat-stop and the ambient temperature will increase, forming a temperature gradient, thus forming the seeing effect at the heat-stop, and introducing wave-front aberration, deteriorating

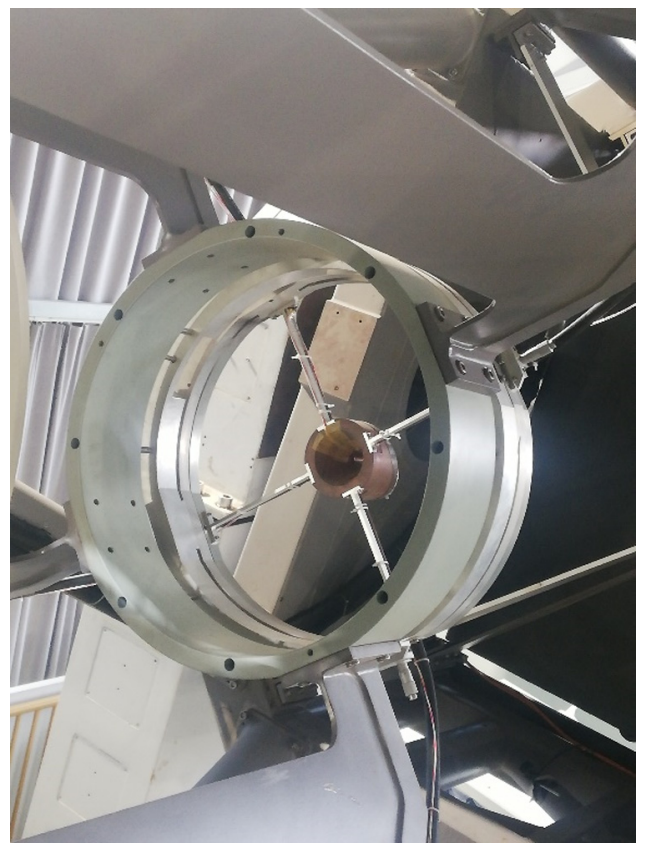

Fig. 4 Structure of the heat-stop for CLST. 
the imaging quality, and reducing the observation efficiency of the telescope ${ }^{4,19}$ In serious cases, the surface of heat-stop will be permanently deformed and damaged. Therefore, the heat-stop must be actively temperature controlled.

The purpose of the whole control system is to control the surface temperature of the heat-stop so the temperature difference between the heat-stop and the environment is as small as possible (the ideal value is $0^{\circ} \mathrm{C}$ ). In CLST, the temperature difference required by the design is $\pm 5^{\circ} \mathrm{C} .{ }^{4}$ Therefore, it is necessary to control the temperature and flow rate at the inlet of the heat-stop. To accurately control the temperature at the inlet of the heat-stop, the outlet temperature of the refrigerator and the power of the electrical heater must be accurately controlled. At the same time, the opening of the electrical control valve should be adjusted according to the temperature difference between the surface temperature of the heat-stop and the ambient temperature, so as to realize the flow control. The core is to establish the mathematical model of the heat-stop. Only when the model is accurate, we can design the control strategy.

To establish the accurate mathematical model of heat-stop, the following assumptions should be made: (1) the flow is two-dimensional flow; (2) the coolant is an incompressible Newton fluid; (3) the physical properties of the coolant do not change during the flow process; (4) the heat dissipation caused by viscous dissipation can be ignored; (5) for spider supporting, the contact area is small, and the location is far away the reflective surface. Thermal conduction by the spider supporting the HS can be neglected when temperature difference is smaller than the required value; and (6) thermal conduction of heat-stop body including reflective structure is very high, and the thickness of these structures is very thin $(<6 \mathrm{~mm})$. Thus, the thermal transmission is fast, and almost all of them are conducted into the cooled coolant.

First of all, we need to consider the main factors affecting the surface temperature of the heatstop. When the heat-stop of the solar telescope is placed in an open environment, the main influencing factors are ambient temperature, local wind speed, solar radiation power, and temperature and flow rate of coolant at the inlet of heat-stop. Under the condition of ignoring the influence of wind speed, as shown in Fig. 5.

According to the law of conservation of energy, the following equation can be formulated:

$$
m C_{p} \frac{d T}{d t}=\gamma Q_{s} A_{1}-h_{2} A_{2}(T-\bar{T})-h_{3} A_{3}\left(T-T_{a}\right)-\varepsilon A_{4} \sigma\left(T^{4}-T_{a}^{4}\right),
$$

where $m$ is the mass of the heat-stop, $\mathrm{kg} ; C_{p}$ is the specific heat capacity of copper alloy, $\mathrm{J} /(\mathrm{kg} \cdot \mathrm{K}) ; \gamma$ is the surface absorptivity of the heat-stop; $Q_{s}$ is the power of solar radiation, $\mathrm{W} / \mathrm{m}^{2} ; A_{1}$ is the surface area of the primary mirror of the facing the sun, $\mathrm{m}^{2} ; h_{2}$ is the heat transfer coefficient between the coolant and the heat-stop, $\mathrm{W} /\left(\mathrm{m}^{2} \cdot \mathrm{K}\right) ; A_{2}$ is the area of the contact surface between the coolant and the heat-stop, $\mathrm{m}^{2} ; \bar{T}$ is the average temperature at the inlet and outlet of the heat-stop, $\bar{T}=\frac{T_{\text {in }}+T_{\text {out }}}{2},{ }^{\circ} \mathrm{C} ; T_{\text {in }}$ is the temperature at the inlet of the heat-stop, ${ }^{\circ} \mathrm{C} ; T_{\text {out }}$ is the temperature at the outlet of the heat-stop, ${ }^{\circ} \mathrm{C} ; h_{3}$ is the heat transfer coefficient between the surface of the heat-stop and the ambient environment, $\mathrm{W} /\left(\mathrm{m}^{2} \cdot \mathrm{K}\right) ; A_{3}$ is the surface area of the heat-stop in contact with the surrounding environment, $\mathrm{m}^{2} ; \varepsilon$ is a radiative property of the surface termed the emissivity, $0 \leq \varepsilon \leq 1 ; \sigma$ is the Stefan-Boltzmann constant $\left[\sigma=5.67 \times 10^{-8} \mathrm{~W} /\left(\mathrm{m}^{2} \cdot \mathrm{K}^{4}\right)\right] ; A_{4}$ is the surface area of the heat-stop in contact with the

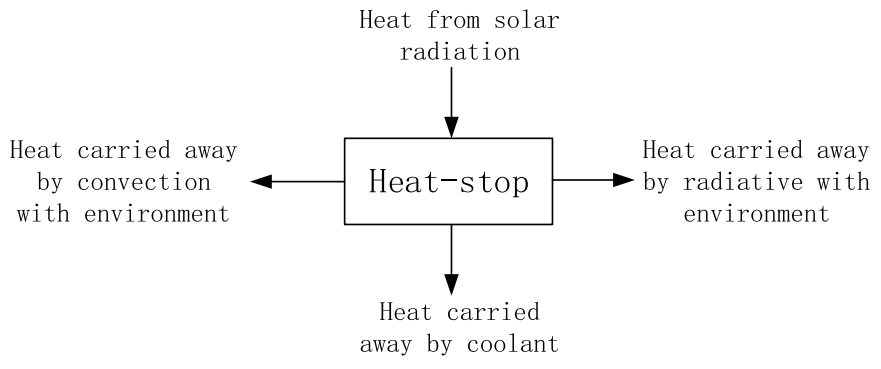

Fig. 5 Main factors affecting the surface temperature of heat-stop. 
surrounding environment, $\mathrm{m}^{2} ; T_{a}$ is the ambient temperature, ${ }^{\circ} \mathrm{C} . ;$ and $T$ is the surface temperature of the heat-stop, ${ }^{\circ} \mathrm{C}$.

The goal of heat-stop thermal control is to ensure that the surface temperature of heat-stop is consistent with the ambient temperature, that is, the temperature difference is zero. Actually, the temperature difference between $T$ and $T_{a}$ should be small for a successful thermal control system. Thus, the time derivative of the temperature at left-hand side should also be small although $\left(T-T_{a}\right)$ is not always zero. When the temperature difference is small and $\sigma=5.67 \times 10^{-8} \mathrm{~W} /\left(\mathrm{m}^{2} \cdot \mathrm{K}^{4}\right)$, the energy term $\varepsilon A_{4} \sigma\left(T^{4}-T_{a}^{4}\right)$ is very small. The energy due to the thermal radiation can be ignored, and Eq. (1) can be rewritten as

$$
m C_{p} \frac{d T}{d t}=\gamma Q_{s} A_{1}-h_{2} A_{2}(T-\bar{T})-h_{3} A_{3}\left(T-T_{a}\right) .
$$

The energy taken away by the coolant can also be expressed as

$$
\Phi=\dot{m}_{\text {cool }} C_{\text {cool }}\left(T_{\text {out }}-T_{\text {in }}\right)=h_{2} A_{2}\left(T-\frac{T_{\text {out }}+T_{\text {in }}}{2}\right),
$$

where $\dot{m}_{\text {cool }}$ is the mass flow of coolant and $C_{\text {cool }}$ is the specific heat capacity of coolant:

$$
\begin{gathered}
T_{\text {out }}=\frac{h_{2} A_{2}}{\dot{m}_{\mathrm{cool}} C_{\mathrm{cool}}+\frac{h_{2} A_{2}}{2} T-\frac{\frac{h_{2} A_{2}}{2}-\dot{m}_{\mathrm{cool}} C_{\mathrm{cool}}}{\dot{m}_{\mathrm{cool}} C_{\mathrm{cool}}+\frac{h_{2} A_{2}}{2}} T_{\mathrm{in}},} \\
\Phi=\dot{m}_{\mathrm{cool}} C_{\mathrm{cool}} \frac{h_{2} A_{2}}{\dot{m}_{\mathrm{cool}} C_{\mathrm{cool}}+\frac{h_{2} A_{2}}{2}}\left(T-T_{\mathrm{in}}\right) .
\end{gathered}
$$

Equation (6) can be obtained by substituting Eq. (5) into Eq. (2):

$$
\begin{aligned}
\frac{d T}{d t}= & -\frac{\left(\dot{m}_{\mathrm{cool}} C_{\mathrm{cool}} \frac{h_{2} A_{2}}{\dot{m}_{\mathrm{cool}} C_{\mathrm{cool}}+\frac{h_{2} A_{2}}{2}}+h_{3} A_{3}\right)}{m C_{p}} T+\frac{\dot{m}_{\mathrm{cool}} C_{\mathrm{cool}} \frac{h_{2} A_{2}}{\dot{m}_{\mathrm{cool}} C_{\mathrm{cool}}+\frac{h_{2} A_{2}}{2}}}{m C_{p}} T_{\text {in }} \\
& +\frac{\gamma A_{1}}{m C_{p}} Q_{s}+\frac{h_{3} A_{3}}{m C_{p}} T_{a} .
\end{aligned}
$$

The actual system parameter settings are shown in Table 1.

The input quantity is $T_{\text {in }}, T_{a}$ and $Q_{s}$. The output quantity is $T$.

The CLST locates in an enclosed dome in Chengdu City, and almost the environment wind is shielded even in operation. In this situation, the convective heat transfer coefficient can be approximately taken as $h_{3}=5 \mathrm{~W} /\left(\mathrm{m}^{2} \cdot \mathrm{K}\right)$ for simplicity. Because the heat exchange between the coolant and the surface of the heat-stop can take away the solar radiation absorbed by the heat-stop, there are two models to calculate the heat transfer coefficient $h_{2}$ : one is the flow along flat plate model, and the other is the jet impingement model.

In CLST ground-based solar telescope, the cooling water is cooled by jet impingement, which is an effective thermal control method. ${ }^{24,25}$ Under the effect of pressure difference, the coolant is injected vertically to the inner surface of the heat-stop through the circular nozzle inside the heat-stop to actively control the temperature of the heat-stop. First, the solar radiation energy absorbed by the heat-stop is transmitted to the inner surface by heat conduction, and then the heat is exchanged with the coolant. ${ }^{17-20}$ Due to the concentration of energy at the heat-stop and the high temperature at the local focus, the jet impingement model can improve the heat transfer coefficient and increase the heat transfer. In CLST, there are two nozzles to ensure uniformity of the heat-transfer coefficient. In fact, if a nozzle array including several nozzles is employed, the thermal uniformity will be better, but the structure of heat-stop will be more complex. In our previous work, ${ }^{3,4}$ a series of CFD simulations have been done to evaluate the thermal 
Table 1 Parameter values of theoretical model of heat-stop.

\begin{tabular}{lcc}
\hline \hline Items & Values & Units \\
\hline$m$ & 1.5 & $\mathrm{~kg}$ \\
$C_{p}$ & 386 & $\mathrm{~J} /(\mathrm{kg} \cdot \mathrm{K})$ \\
$\gamma$ & 0.05 & - \\
$A_{1}$ & 2.54 & $\mathrm{~m}^{2}$ \\
$A_{3}$ & $3.15 \times 10^{-2}$ & $\mathrm{~m}^{2}$ \\
$h_{3}$ & 5 & $\mathrm{~W} /\left(\mathrm{m}^{2} \cdot \mathrm{K}\right)$ \\
$h_{2}$ & 11,000 & $\mathrm{~W} /\left(\mathrm{m}^{2} \cdot \mathrm{K}\right)$ \\
$A_{2}$ & $3.5 \times 10^{-3}$ & $\mathrm{~m}{ }^{2}$ \\
$\dot{m}_{\text {cool }}$ & 0.015 & $\mathrm{~kg} / \mathrm{s}$ \\
$C_{\text {cool }}$ & 4160 & $\mathrm{~J} /(\mathrm{kg} \cdot \mathrm{K})$ \\
\hline \hline
\end{tabular}

efficiency for different number of nozzles, and two inlets are the most optimizing result by balancing the efficiency and complexity. In our mathematical model, we replace two inlets as a single nozzle to acquire equivalent thermal efficiency for simplicity.

The value of $h_{2}$ is related to the flow rate of the coolant, the geometric size of the heat-stop, and the thermos-physical parameters. In the circle with the stagnation point as the center and radius as $r$, the average heat transfer coefficient of the impacted surface can be written as ${ }^{26}$

$$
\frac{h_{m} D}{\lambda}=\left(N u_{D}\right)_{m}=f\left(\frac{H}{D}, \frac{r}{D}, \operatorname{Re}_{D}, P r\right) .
$$

The formula of Gnielinski is used as Eq. (4): ${ }^{26}$

$$
N u_{f}=\frac{(f / 8)(\operatorname{Re}-1000) \operatorname{Pr}_{f}}{1+12.7 \sqrt{f / 8}\left(\operatorname{Pr}_{f}^{2 / 3}-1\right)}\left[1+\left(\frac{d}{l}\right)^{2 / 3}\right] c_{t}
$$

For noncircular cross-section, the equivalent diameter $d_{e}$ can be used as the characteristic scale. For the nonstraight pipes, the calculated average $N u$ number is multiplied by a correction coefficient $c_{r}$. For liquids, $c_{r}=1+10.3\left(\frac{d_{e}}{R}\right)^{3}$ is taken. $^{26}$

The Reynolds number $\operatorname{Re}=\frac{u d}{\nu}$ can be determined according to the flow rate of the coolant. By looking up the physical parameters of the coolant, it can be found that the Prandtl number $\operatorname{Pr}=\frac{\mu C_{p}}{\lambda}$ and the resistance coefficient $f=[1.82 \times \lg (\mathrm{Re})-1.64]^{-2} \cdot{ }^{26}$ According to the above approximate Eqs. (7) and (8), the average heat transfer coefficient $h_{2}=h_{m}=11,000 \mathrm{~W} /\left(\mathrm{m}^{2} \cdot \mathrm{K}\right)$ can be approximately calculated. Besides that, the position of the virtual single nozzle will not change calculating results of our mathematical model, although the uniformity will be affected. Thus, $h_{2}=11,000 \mathrm{~W} /\left(\mathrm{m}^{2} \cdot \mathrm{K}\right)$ is also an equivalent thermal transmission efficiency.

\section{Experiment Validation of Heat-Stop Model}

The heat-stop thermal control system is based on the 1.8-m ground-based solar telescope project of Institute of Optics and Electronics, Chinese Academy of Sciences. The schematic diagram of the experimental device is shown in Fig. 6.

Figure 7 shows the ground-based solar telescope, which is pointing to the sun for CLST. The experimental device corresponding to the schematic diagram is shown in Fig. 8. Figure 9 shows the position of solar power meter for CLST. 


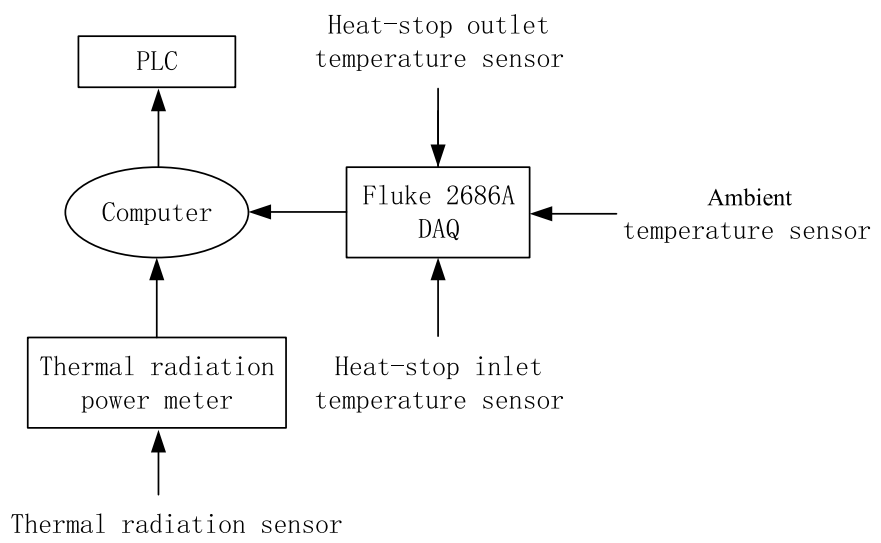

Fig. 6 Schematic diagram of the experimental device.

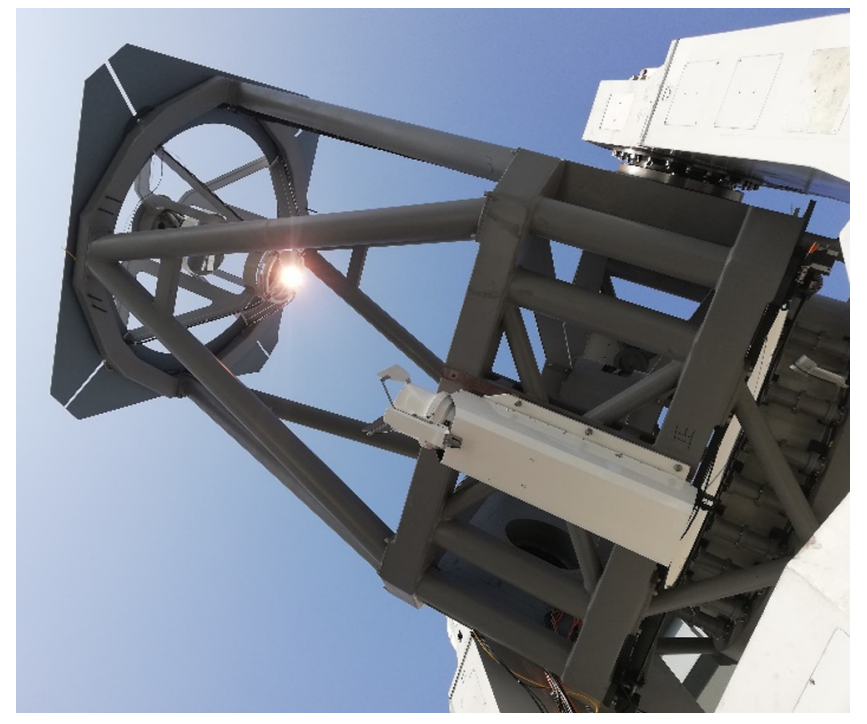

Fig. 7 In observation, which is pointing to the sun for CLST.

Six temperature sensors in total are adopted in our experiment. To accurately measure the surface temperature of the heat-stop, temperature sensor, whose accuracy is better than $\pm 2^{\circ} \mathrm{C}$, is installed on the inner conical surface of the heat-stop near the through hole. The chip of temperature sensor is pasted by a thin layer of silica gel with high thermal conduction. Meanwhile, the outer faces of the temperature sensor have been covered by a kind of thermal insulation material. To measure the temperature at the inlet and outlet of the heat-stop, four temperature sensors were installed at the inlet and outlet of the heat-stop substrate, and a solar radiation power was installed on the sunshade of the ground-based solar telescope that can measure the solar irradiance in real time. A fluke temperature sensor with high precision is installed near the telescope structure where the light path is not affected, and the ventilation position is used to measure the ambient temperature. These temperature sensors are collected by fluke data acquisition $2686 \mathrm{~A}$, which is $0.5 \mathrm{~Hz}$ sampling and the temperature is recorded by corresponding software in real-time.

After a period of operation (about $20 \mathrm{~min}$ ), the difference between the surface temperature of the heat-stop and the ambient temperature is within $\pm 5^{\circ} \mathrm{C}$. At this time, observe and record the corresponding experimental data, mainly the measured temperature and solar irradiance of each sensor.

On July 2, 2020, we carried out the relevant experimental verification work. In the experiments, the coolant flow rate is $15 \mathrm{ml} / \mathrm{s}$, and PI control algorithm is used to keep the outlet temperature of the refrigerator constant at $7^{\circ} \mathrm{C} .7^{\circ} \mathrm{C}$ is defined on the basis of the ambient 


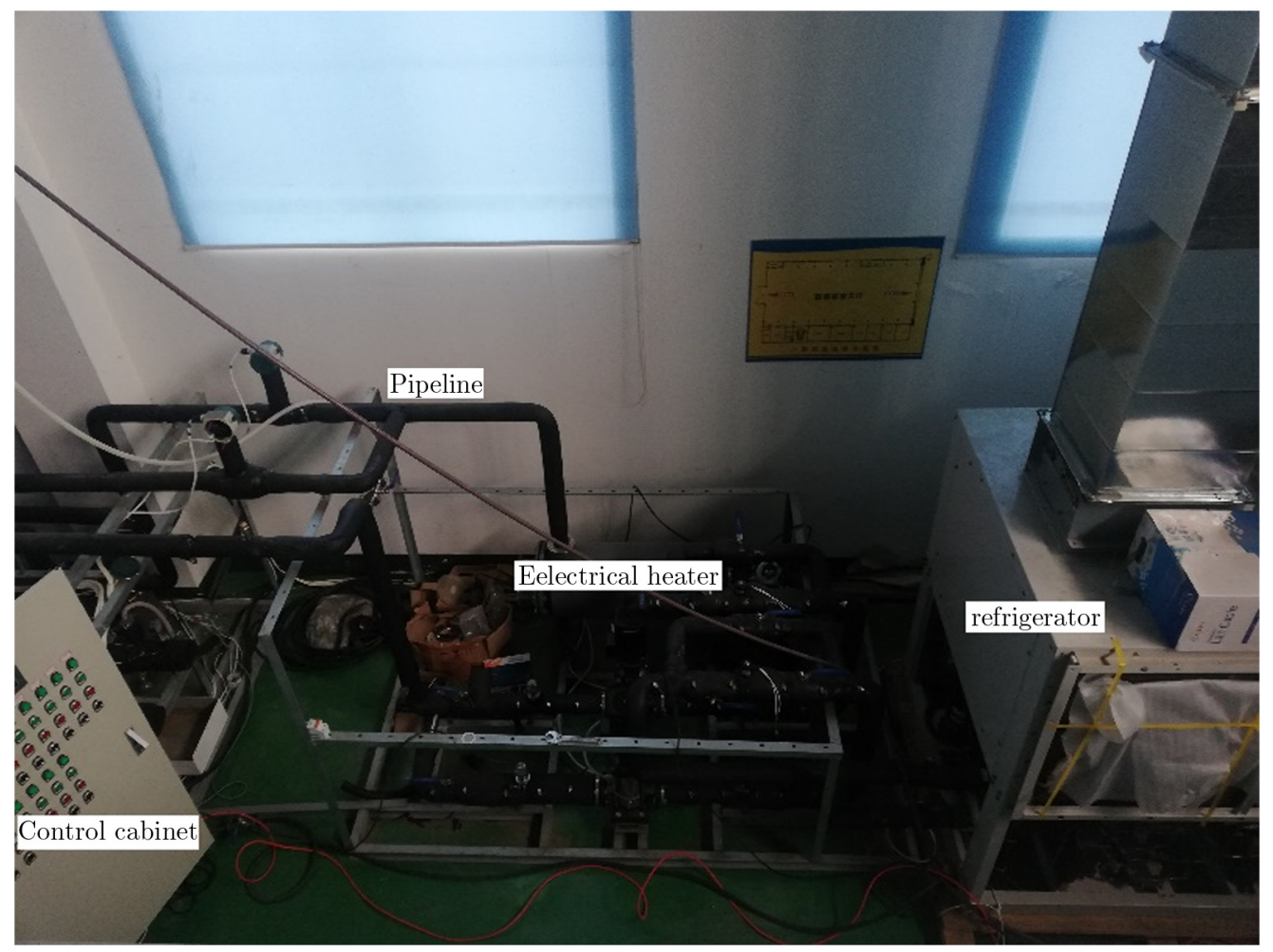

Fig. 8 Experimental device of active temperature control system for CLST.

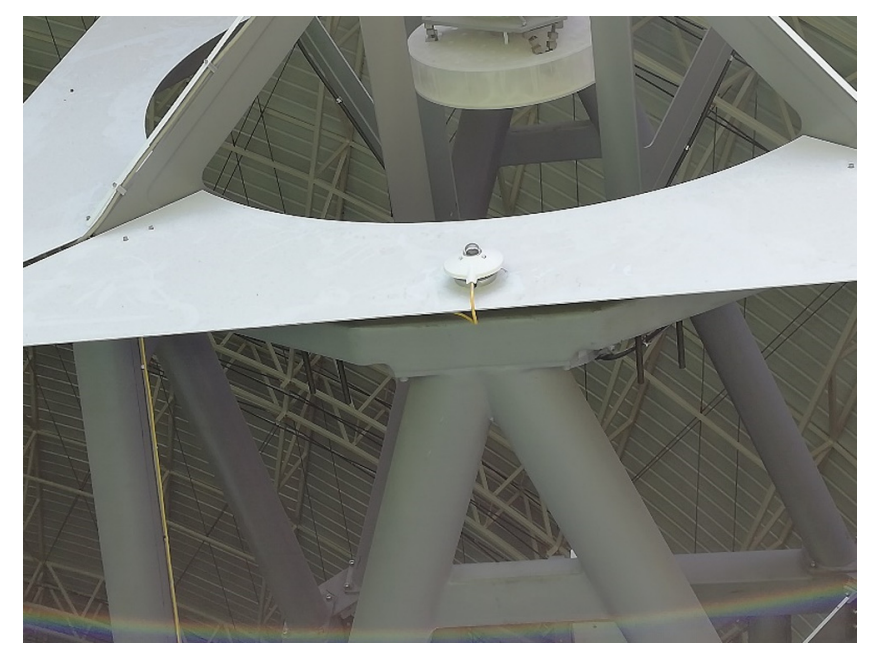

Fig. 9 Position of solar power meter for CLST.

temperature, which depends on the performance of the used refrigerator. As the input signals, the measured heat-stop inlet temperature, outlet temperature, ambient temperature, and solar irradiance curves are shown in Fig. 10. Under these inputs/outputs, the surface temperature of the heat-stop calculated by the theoretical model and the experimental value is shown in Fig. 11, and the difference curve is shown in Fig. 12.

In general, the change of ambient temperature is lagging of the sun radiation change. Thus, it is possible that the temperature is rising when the solar radiation is decreasing. It can be seen from Fig. 12 that there is little difference between the theoretical value and the experimental value. In the range of $\pm 2{ }^{\circ} \mathrm{C}$, this is mainly due to some assumptions made in the process of model approximation. (1) In the theoretical calculation, the influence of wind speed is ignored, 
Yan et al.: Modeling and experiment validation of thermal control for the heat-stop...
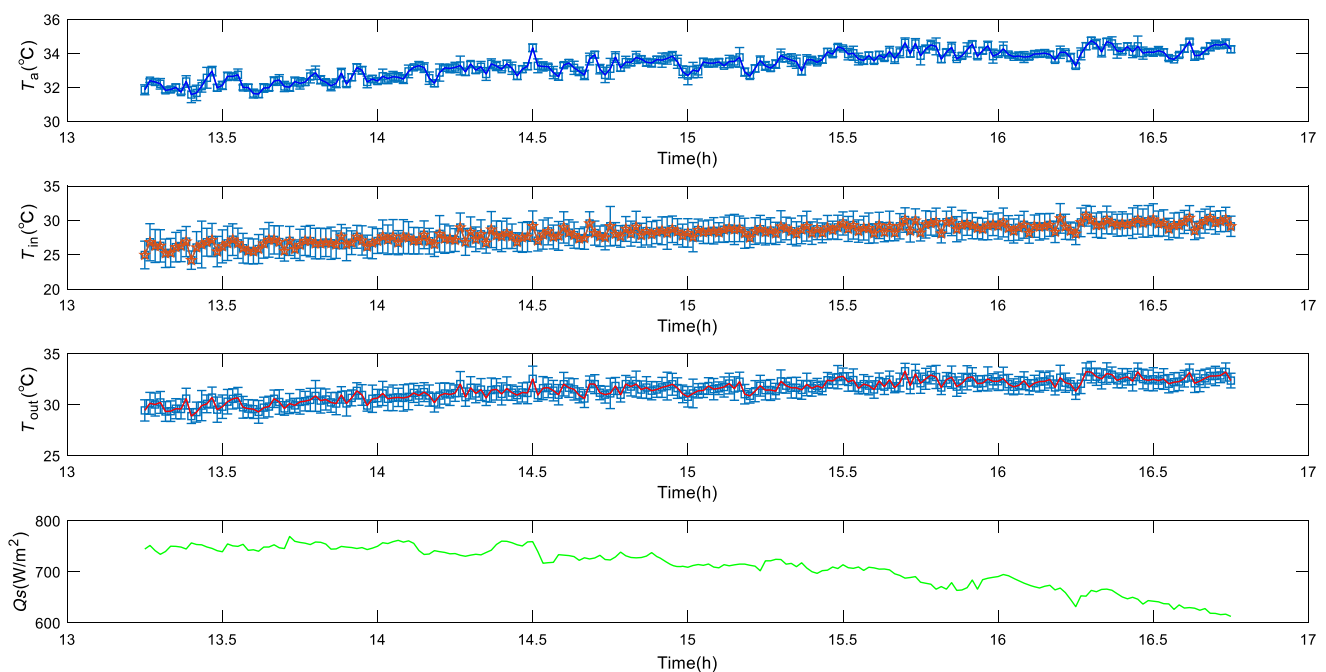

Fig. 10 Curves of the input/output signals.
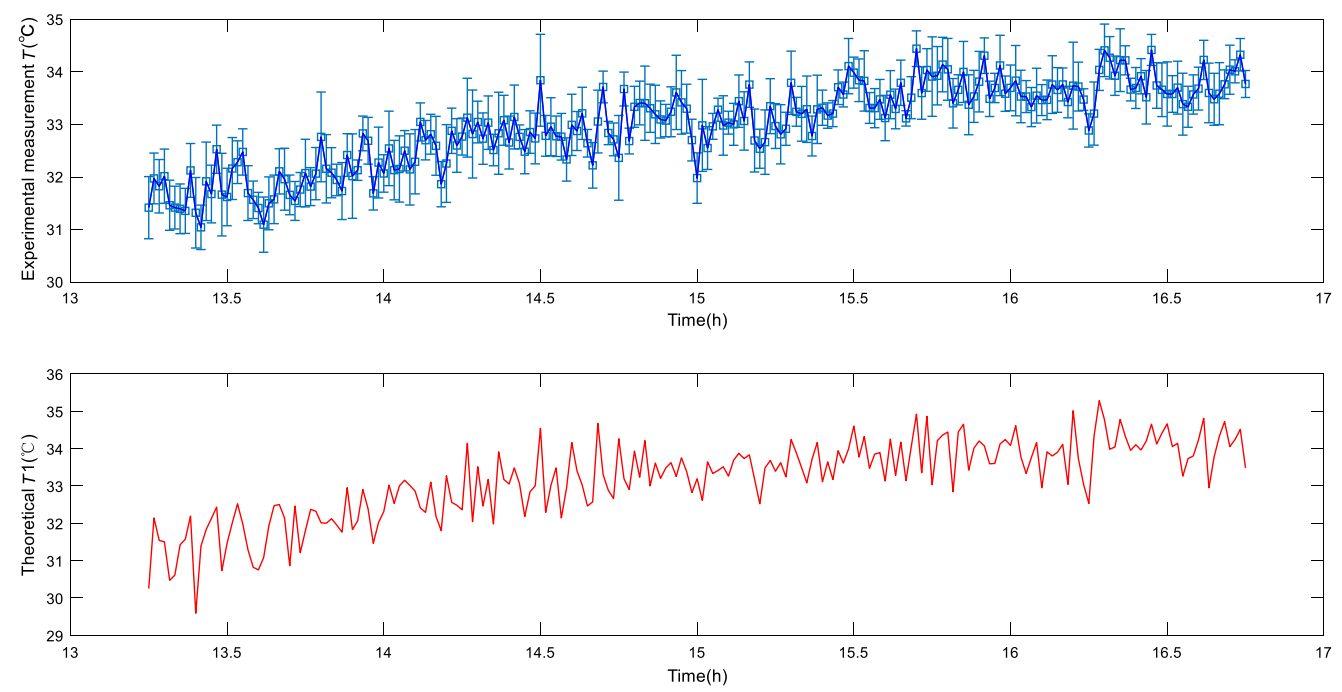

Fig. 11 Theoretical $T_{1}$ and experimental measurements $T$ of heat-stop surface temperature.

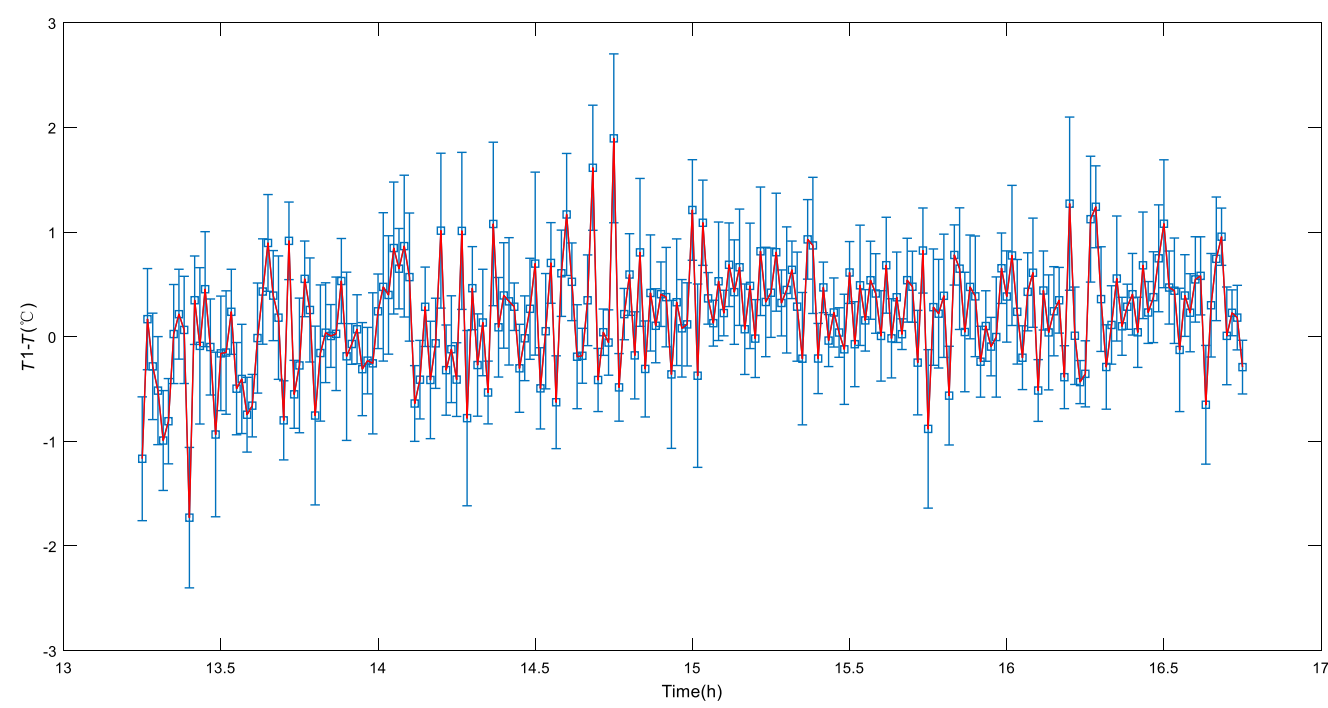

Fig. 12 Temperature difference curve between the theoretical value and experimental value. 

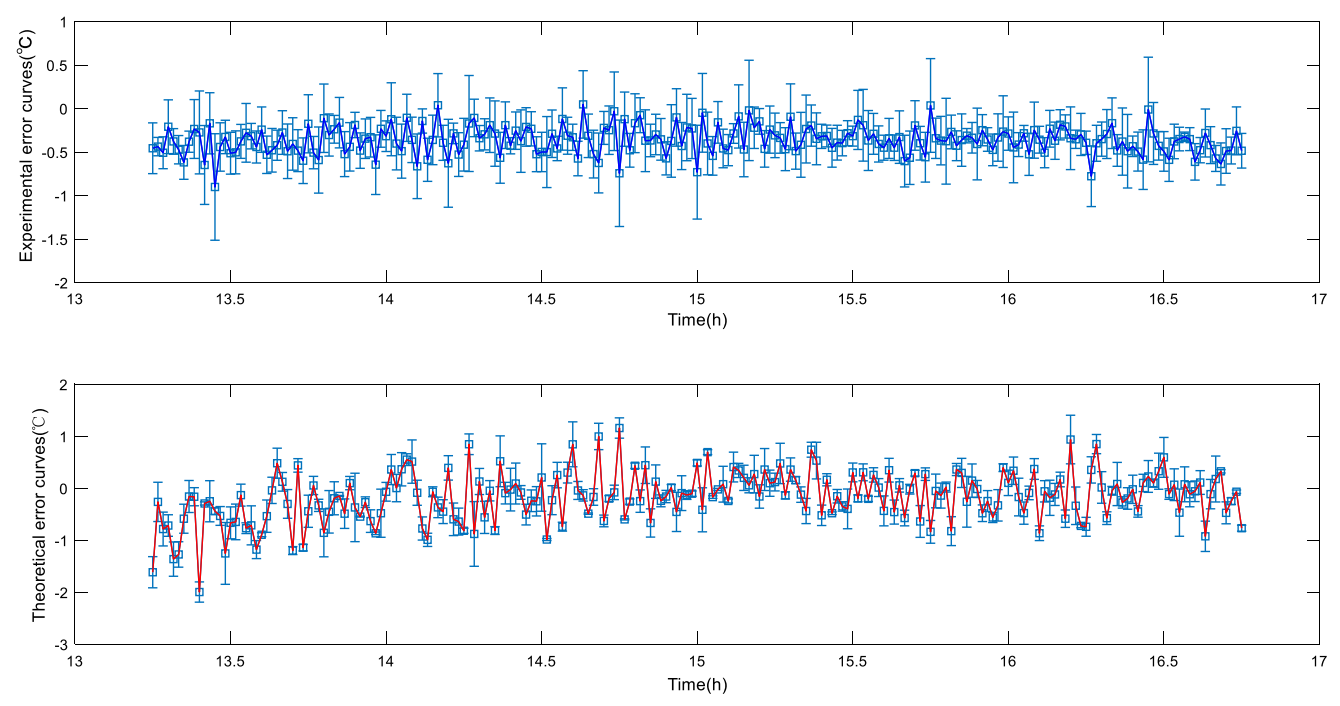

Fig. 13 Theoretical error curves and experimental error curves between the heat-stop surface temperature and ambient temperature.

which leads to the difference of convective heat transfer coefficient between heat-stop and environment; (2) due to the asymmetry of heat-stop structure, the calculation of heat transfer coefficient between coolant and heat exchanger is approximate in the process of fluid structure coupling; (3) in the theoretical calculation, the thermal radiation term between heat-stop and environment is ignored, which leads to certain difference.

In the theoretical calculation, the temperature difference curve between the surface temperature of the heat-stop and the ambient temperature is shown in Fig. 13. The maximum temperature difference is $2.0^{\circ} \mathrm{C}$, which meets the temperature control target that the surface temperature of the heat-stop does not exceed the ambient temperature by $5^{\circ} \mathrm{C}$. In the experiment, the temperature difference curve between the surface temperature of the heat-stop and the ambient temperature is shown in Fig. 13, and the maximum temperature difference is $2^{\circ} \mathrm{C}$, which also meets the temperature control target that the surface temperature of the heat-stop does not exceed the ambient temperature by $5^{\circ} \mathrm{C}$. Therefore, it can be verified that the reflective heat-stop model established by the law of conservation of energy is consistent with the experimental situation and the model is correct.

\section{Conclusions}

Thermal control system is one of the important systems to ensure the normal operation of large ground-based solar telescope. The heat-stop at the telescope's primary focus plays a decisive role in thermal control system. Typically, the size of the through hole in the heat-stop is defined by the demands of the telescope optics. It can limit the field of view of the follow-up optical system and protect the subsequent optical system. The mathematical model of heat-stop has a great influence on the selection of control strategy, that is, the selection of control parameters.

In this paper, we propose an analytical model for the estimation of the surface temperature of the HS of CLST. The feasibility of the method is verified by the corresponding experiments. The experimental results show that the modeling process is correct. The experimental results are in good agreement with the theoretical results and within the allowable error range. The model can be used to determine the control parameters of the follow-up control strategy and has certain application value.

\section{Acknowledgments}

We would like to thank Mr. Lin Kong, Dr. Jiawei Yong, Dr. Yunfei Wu, Dr. Jingwen Zhang, et al. at the Institute of Optics and Electronics (IOE), Chinese Academy of Science (CAS), for their 
cooperation and important help. We also appreciate the reviewers for their careful revisions and constructive comments, which helped to improve the quality of this paper. This research was funded by the National Natural Science Foundation of China, Grant Nos. 11643008, 11727805, 61905252, and 12022308, and by Youth Innovation Promotion Association, Chinese Academy of Science Grant No. 2018412.

\section{References}

1. R. Schlichenmaier et al., "Science requirement document (SRD) for the European Solar Telescope (EST), (2nd edition, December 2019)" (2019).

2. M. P. Rast et al., "Critical Science Plan for the Daniel K. Inouye Solar Telescope (DKIST)," Sol. Phys. 296, 70 (2021).

3. Y. Liu, "Active thermal control technology for large ground-based solar telescope," The Chinese Academy of Sciences, Beijing (2016).

4. Y. Liu, N. Gu, and C. Rao, "Quantitative evaluation on internal seeing induced by heat-stop of solar telescope," Opt. Express 23(15), 19980-19995 (2015).

5. A. P. Verdoni and C. Denker, "The thermal control of the new solar telescope at Big Bear Observatory," Proc. SPIE 6267, 62670M (2006).

6. G. Murga et al., "DKIST enclosure fabrication factory assembly and testing" (2014).

7. S. Berukoff, K. Reardon, and T. Rimmele, "Next-generation solar data and data services from the Daniel K. Inouye Solar Telescope," Astron. Data Anal. Softw. Syst.: XXIV ASP Conf. Ser. 495, 91-94 (2015).

8. C. R. White and L. Phelps, "DKIST facility management system integration," Proc. SPIE 9906, 990652 (2016).

9. A. Tritschler et al., "Daniel K. Inouye Solar Telescope: high-resolution observing of the dynamic Sun," Astron. Nachr. 337(10), 1064 (2016).

10. R. Volkmer, "Thermal characteristics of the solar telescope GREGOR," Proc. SPIE 7012, 70120K (2008).

11. R. Volkmer et al., "GREGOR telescope-start of commissioning," Proc. SPIE 7733, $77330 \mathrm{~K}(2010)$.

12. R. Volkmer et al., "Optical and thermal design of the main optic of the solar telescope GREGOR," Proc. SPIE 5179, 77330K (2003).

13. F. Kneer et al., "GREGOR, a 1.5 m Gregory-type telescope for solar observation," Il Nuovo Cimento C 025(5-6), 689 (2002).

14. F. Berrilli et al., "The heat stop for the 4-m European Solar Telescope EST," Proc. SPIE 7733, $77332 Z$ (2010).

15. T. Berkefeld et al., "Site-seeing measurements for the European Solar Telescope," Proc. SPIE 7733, 77334I (2010).

16. M. Collados, "European Solar Telescope (EST): project status," Proc. SPIE 7012, 70120J (2008).

17. C. Rao et al., "1.8-m solar telescope in China: Chinese Large Solar Telescope," J. Astron. Telesc. Instrum. Syst. 1(2), 024001 (2015).

18. N. Gu et al., "Thermal control for light-weighted primary mirrors of large ground-based solar telescopes," J. Astron. Telesc. Instrum. Syst. 5(1), 014005 (2019).

19. N. Gu et al., "Passive control of the temperature homogeneity for the primary mirror surface of large ground-based solar telescopes," J. Astron. Telesc. Instrum. Syst. 5(4), 044007 (2019).

20. Y. Liu et al., "Heat-stop structure design with high cooling efficiency for large ground-based solar telescope," Appl. Opt. 54(21), 6441-6447 (2015).

21. C. Rao et al., "First light of the 1.8-m solar telescope-CLST," Science China Physics, Mechanics and Astronomy 63(10), 109631 (2020).

22. T. Kosugi et al., "The Hinode (Solar-B) mission: an overview," Sol. Phys. 243(1), 3-17 (2007).

23. F. Berrilli et al., "ADAHELI+: exploring the fast, dynamic Sun in the x-ray, optical, and near-infrared," J. Astron. Telesc. Instrum. Syst. 1, 044006 (2015). 
24. P. Culun, N. Celik, and K. Pihtili, "Effects of design parameters on a multi jet impinging heat transfer," AEJ -Alexandria Engineering Journal 57(4), 4255-4266 (2018).

25. S. V. Narumanchi et al., "Comparison of different single-phase liquid jet impingement cooling configurations in the context of thermal management in power electronics," in ASME Pacific Rim Technical Conf. and Exhib. Integr. and Packaging of MEMS (2005).

26. T. Wenquan, Heat Transfer, 5th ed., Higher Education Press, Beijing (2019).

Zhiwu Yan is a PhD candidate at the Institute of Optics and Electronics, Chinese Academy of Science (CAS). He received his BS degrees in mechatronic engineering from Beijing Institute of Petro-chemical Technology in 2002, and he received his MS degrees in measurement technology and automatic equipment from Beijing University of Aeronautics and Astronautics in 2005. His current research interest is the design of thermal control system for ground-based telescope.

Naiting Gu is a professor at the Institute of Optics Electronics (IOE), CAS. He received his BS degree in optics from Chongqing University in 2007 and his $\mathrm{PhD}$ in optical engineering from the University of CAS in 2012. He is the author of more than 30 journal papers and has written one book chapter. His current research interests include solar telescope systems, interferometry, polarization imaging, thermal control, and adaptive optics systems.

Yangyi Liu is an associate professor at the Sichuan Police College. He received his BS degree in mechanical engineering and automation from Chongqing University in 2012 and his $\mathrm{PhD}$ in optical engineering from the University of CAS in 2016. His research interests include thermal control technology of solar telescopes.

Youming Guo is currently an associate professor in the Institute of Optics and Electronics (IOE), CAS. He received his BS degree in the Department of Automation, University of Science and Technology of China in 2009 and his PhD in signal and information processing from the University of CAS in 2014. His current research interests include adaptive optics technology, specifically involving the application of advanced control algorithm and machine learning technology in adaptive optics system.

Changhui Rao is a professor at IOE, CAS. He received his BS degree in optical metrology from Wuhan University in 1993 and his $\mathrm{PhD}$ in optical engineering from the University of CAS in 2001. He has researched on adaptive optics technology for over 20 years. He is the author of more than 200 journal papers and has written two book chapters. His current research interests include solar telescope systems, adaptive optics, and solar observation with high resolution. 